\title{
Slowed Relaxational Dynamics Beyond the Fluctuation-Dissipation Theorem
}

\author{
P. De Gregorio ${ }^{\mathrm{a}, \mathrm{b}}$, F. Sciortino ${ }^{\mathrm{b}}$, P. Tartaglia ${ }^{\mathrm{b}}$, E. Zaccarelli ${ }^{\mathrm{a}}$ \\ and K. A. Dawson ${ }^{a}$ \\ ${ }^{a}$ Irish Centre for Colloid Science and Biomaterials, Department of Chemistry, \\ University College Dublin, Belfield, Dublin 4, Ireland \\ ${ }^{\mathrm{b}}$ Dipartimento di Fisica Università di Roma La Sapienza, Istituto Nazionale di \\ Fisica della Materia, and INFM Center for Statistical Mechanics and Complexity \\ Piazzale Aldo Moro 2, 00185 Roma, Italy
}

\begin{abstract}
To describe the slow dynamics of a system out of equilibrium, but close to a dynamical arrest, we generalize the ideas of previous work to the case where timetranslational invariance is broken. We introduce a model of the dynamics that is reasonably general, and show how all of the unknown parameters of this model may be related to the observables or to averages of the noise. One result is a generalisation of the Fluctuation Dissipation Theorem of type two (FDT2), and the method is thereby freed from this constraint. Significantly, a systematic means of implementing the theory to higher order is outlined. We propose the simplest possible closure of these generalized equations, following the same type of approximations that have been long known for the equilibrium case of Mode Coupling Theory (MCT). Naturally, equilibrium MCT equations are found as a limit of this generalized formalism.
\end{abstract}

Key words: Fluctuation-Dissipation Theorem, glasses, Mode Coupling Theory, aging

PACS: 61.20.Lc, 64.70.Pf, 05.20-y

\section{Introduction}

Glasses are typically amorphous, dynamically slowed or arrested states of matter, whose dynamical relaxational processes are dramatically slower than those of liquid states, despite their great structural similarities. It has long been known that, for many systems, slow and careful approach to what is clearly a non-equilibrium glass transition leads to entirely reproducible behaviour more reminiscent of phase transitions at equilibrium. Such phenomena are often 
called, albeit loosely, 'equilibrium glass transitions'. The most complete current microscopic theory available for these systems, Mode Coupling Theory (MCT) [1] has implicit within it [2] the Fluctuation Dissipation Theorem of type two (FDT2) [3], but despite this, the agreement between theory and light scattering measurements [4] are remarkably good for simple colloidal systems. The explicit manifestation of the 'equilibrium' nature of the system is that all observables are functions only of the time differences, and are independent of when the experimental measurements are commenced. However, cases such as this are by no means natural, and typically experiments are found to depend on the time of waiting $t_{w}$ after a change in the external parameters of the system. Numerous experimental descriptions exist. However, in a pioneering paper [5] on model systems it was shown that equilibrium MCT ideas could be extended to deal with systems that 'age' and therefore violate FDT2 [6-8]. The ideas contained in that paper have been helpful in developing the concept of aging, but it is not until recently that attempts have been made to extend the existing microscopic theory of equilibrium glassy states (MCT) in the same spirit. Two such approaches appear to be developing. One of these is described in this paper. The other [9] uses an entirely different strategy by which projection operators are extended to the non-equilibrium state.

Before beginning our discussion, we should sound some notes of caution. Whilst the framework we build is quite general, the approximations we use are the analogues of those used in equilibrium MCT [1], and in non-microscopic theories of aging [5], [10]-[13]. We have argued elsewhere [2,14] that these amount to a type of dynamical approximation, and that is known to be accurate close to the arrest transition only for colloidal systems. Elsewhere, as in molecular glasses, they are approximately correct up to some characteristic distance from the glass transition, often called the MCT temperature [15]. In terms of approximations, what we here present cannot be expected to improve on this fundamental limitation. On the other hand we may hope that what is described below will be the non-equilibrium (aging) theory of those systems for which MCT has proven of value.

\section{Fundamentals}

It has been shown in previous works $[2,14]$ that it is possible to write density equations of motion starting from Newtons equations. This gives an explicit formula for $\ddot{\rho}_{\mathbf{k}}(t)$ where the density variables $\rho_{\mathbf{k}}(t)$ are defined as the Fourier transform of the number density $\rho(\mathbf{r}, t)$, i.e.

$$
\rho_{\mathbf{k}}(t)=\int_{V} d \mathbf{r} \rho(\mathbf{r}, t) e^{i \mathbf{k} \cdot \mathbf{r}}=\sum_{j=1}^{N} e^{i \mathbf{k} \cdot \mathbf{r}_{j}(t)}
$$


In principle, to describe the underlying Newtonian variables of position and velocity we would need many fields, the most fundamental of which are generally considered to be the hydrodynamic variables of longitudinal and transverse currents, along with a local temperature or entropy variable. Until now, mode coupling type theories have been based on longitudinal current, and therefore only the density degree of freedom, and we shall illustrate our points in the following within this particular approximation. Thus, the first time derivative of density is related to the longitudinal current, and the derivative of this current is treated in Mode Coupling Theory (MCT), leading to an equation involving the second order derivative of density in time [1]. Extensions involving more fields are more complex, and they will be the subject of future work.

The basic approach in any case is to develop a general model that can describe slow out-of-equilibrium dynamics by extending FDT2 [3], and then to find some simple approximations to close the model, as a starting point of applicability.

Thus, we write the density equations as,

$$
\ddot{\rho}_{\mathbf{k}}(t)=\mathcal{D}_{\mathbf{k}}(t, \tau)+\mathcal{R}_{\mathbf{k}}(t, \tau)
$$

where we separate out deterministic and stochastic motions, representing with $\mathcal{D}_{\mathbf{k}}(t, \tau)$ and $\mathcal{R}_{\mathbf{k}}(, \tau)$ respectively the deterministic and the random force acting on the density waves. Observables have to be calculated by averaging over the noise distribution which has not yet been stipulated.

We have explicitly indicated the dependence on two distinct times in the history of the system, $t$ and $\tau$. In general, $t$ can represent the final observation time, while $\tau$ can be considered the so-called waiting time in an aging experiment. The choice of these two times implies that, once the variables of the system are known at time $\tau$, equations (2) determine the dynamics of the density variables through the unknown functions $\mathcal{D}_{\mathbf{k}}(t, \tau)$ and $\mathcal{R}_{\mathbf{k}}(t, \tau)$.

We start by discussing some general properties that the random force must possess. Indeed, it is fundamental to require the stochastic process to satisfy the causality condition. Essentially all that we will show, up to the final approximations, derive only from the form of equations (2), and the causality requirements. These explicitly are,

$$
\begin{aligned}
& \left\langle\rho_{-\mathbf{k}}(\tau) \mathcal{R}_{\mathbf{k}}(t, \tau)\right\rangle=0 \\
& \left\langle\dot{\rho}_{-\mathbf{k}}(\tau) \mathcal{R}_{\mathbf{k}}(t, \tau)\right\rangle=0
\end{aligned}
$$

where $t$ corresponds to all times later than $\tau$. The brackets indicate averages over the (unknown) non-equilibrium distribution of the system. If the decom- 
position into deterministic and stochastic motions, made in (2), was exact, these averages would be the same as the averages over the noise distribution.

Now, using (2), the two causality conditions (3) and (4) can be rewritten as,

$$
\begin{aligned}
& \left\langle\rho_{-\mathbf{k}}(\tau) \ddot{\rho}_{\mathbf{k}}(t)\right\rangle=\left\langle\rho_{-\mathbf{k}}(\tau) \mathcal{D}_{\mathbf{k}}(t, \tau)\right\rangle \\
& \left\langle\dot{\rho}_{-\mathbf{k}}(\tau) \ddot{\rho}_{\mathbf{k}}(t)\right\rangle=\left\langle\dot{\rho}_{-\mathbf{k}}(\tau) \mathcal{D}_{\mathbf{k}}(t, \tau)\right\rangle
\end{aligned}
$$

These also imply two further conditions, that are obtained by taking their first derivative with respect to $\tau$, and then using (3) and (4) as well as (2). Thus, we have,

$$
\begin{gathered}
\left\langle\rho_{-\mathbf{k}}(\tau) \frac{\partial}{\partial \tau} \mathcal{D}_{\mathbf{k}}(t, \tau)\right\rangle=0 \\
\left\langle\dot{\rho}_{-\mathbf{k}}(\tau) \frac{\partial}{\partial \tau} \mathcal{D}_{\mathbf{k}}(t, \tau)\right\rangle=\left\langle\ddot{\rho}_{-\mathbf{k}}(\tau) \mathcal{R}_{\mathbf{k}}(t, \tau)\right\rangle \\
=\left\langle\mathcal{D}_{-\mathbf{k}}(\tau, \tau) \mathcal{R}_{\mathbf{k}}(t, \tau)\right\rangle+\left\langle\mathcal{R}_{-\mathbf{k}}(\tau, \tau) \mathcal{R}_{\mathbf{k}}(t, \tau)\right\rangle
\end{gathered}
$$

Conditions $(7,8)$ constitute two of the fundamental constraints on which to build a theory of non-equilibrium slowed dynamics.

At this point we are still at liberty to use any trial deterministic force. We choose to write the most general linear form, i.e.

$$
\begin{aligned}
\mathcal{D}_{\mathbf{k}}(t, \tau) & =-\Omega_{\mathbf{k}}(t) \rho_{\mathbf{k}}(t)-\nu_{\mathbf{k}}(t) \dot{\rho}_{\mathbf{k}}(t) \\
& -\int_{\tau}^{t} d t^{\prime} \gamma_{\mathbf{k}}\left(t, t^{\prime}\right) \dot{\rho}_{\mathbf{k}}\left(t^{\prime}\right)-\int_{\tau}^{t} d t^{\prime} \chi_{\mathbf{k}}\left(t, t^{\prime}\right) \rho_{\mathbf{k}}\left(t^{\prime}\right)
\end{aligned}
$$

where the quantities $\Omega_{\mathbf{k}}(t), \nu_{\mathbf{k}}(t), \gamma_{\mathbf{k}}\left(t, t^{\prime}\right)$ and $\chi_{\mathbf{k}}\left(t, t^{\prime}\right)$ are four unknown parameters of the theory. We may note that so far, if one accepts the underlying Mori-type hypothesis that dynamics may be represented by a deterministic (slow) part, and noise (fast) part, even to describe non-equilibrium states, the linear approximation could be essentially exact for most conditions, providing the memory kernels are chosen correctly.

Substituting (9) into equations (2), we obtain the set of generalised Langevin equations for the density variables as,

$$
\ddot{\rho}_{\mathbf{k}}(t)+\nu_{\mathbf{k}}(t) \dot{\rho}_{\mathbf{k}}(t)+\Omega_{\mathbf{k}}(t) \rho_{\mathbf{k}}(t)
$$




$$
+\int_{\tau}^{t} d t^{\prime} \gamma_{\mathbf{k}}\left(t, t^{\prime}\right) \dot{\rho}_{\mathbf{k}}\left(t^{\prime}\right)+\int_{\tau}^{t} d t^{\prime} \chi_{\mathbf{k}}\left(t, t^{\prime}\right) \rho_{\mathbf{k}}\left(t^{\prime}\right)=\mathcal{R}_{\mathbf{k}}(t, \tau)
$$

With the expression (9), we assume that the deterministic force is composed of linear terms in $\rho_{\mathbf{k}}(t)$ and $\dot{\rho}_{\mathbf{k}}(t)$, explicitly separated into instantaneous and non-local contributions. $\Omega_{\mathbf{k}}(t)$ and $\nu_{\mathbf{k}}(t)$ are time dependent because the system is evolving towards equilibrium and, therefore, so are the collective variables. $\Omega_{\mathbf{k}}(t)$ is the frequency of the elementary excitations that would be 'phonons' at equilibrium, while $\nu_{\mathbf{k}}(t)$ takes into account the dumping of the modes. The integral terms are memory contributions to these kinds of effects, configurational evolution and dissipation respectively. They are not time-translational invariant as the observables of the system are not.

It is not possible to apply a simple FDT2 as soon as this invariance is broken. Nevertheless, the choice made in (9) enforces the width of the noise distribution to be closely related to the memory kernels as was for the equilibrium case. This can be viewed as a generalization of FDT2 for a system out of equilibrium. Indeed, combining $(7,8)$ and $(9)$ we obtain,

$$
\left\langle\mathcal{R}_{-\mathbf{k}}(\tau, \tau) \mathcal{R}_{\mathbf{k}}(t, \tau)\right\rangle=N\left\{J_{k}(\tau)-\frac{1}{4} \frac{\dot{S}_{k}^{2}(\tau)}{S_{k}(\tau)}\right\} \gamma_{\mathbf{k}}(t, \tau)
$$

and

$$
\chi_{\mathbf{k}}(t, \tau)=-\frac{1}{2} \gamma_{\mathbf{k}}(t, \tau) \frac{\partial}{\partial \tau} \log S_{k}(\tau)
$$

with the definitions,

$$
\begin{aligned}
& S_{k}(t, \tau)=\frac{1}{N}\left\langle\rho_{-\mathbf{k}}(\tau) \rho_{\mathbf{k}}(t)\right\rangle \\
& J_{k}(t, \tau)=\frac{1}{N}\left\langle\dot{\rho}_{-\mathbf{k}}(\tau) \dot{\rho}_{\mathbf{k}}(t)\right\rangle \\
& S_{k}(\tau)=S_{k}(\tau, \tau) ; \quad J_{k}(\tau)=J_{k}(\tau, \tau)
\end{aligned}
$$

Here, $S_{k}(\tau)$ and $J_{k}(\tau)$ are equal time correlators. In the equilibrium case, clearly, they simply reduce to,

$$
S_{k}^{(e q)}(t)=S_{k} ; \quad J_{k}^{(e q)}(\tau)=\frac{k^{2}}{\beta m}
$$

Also, $\dot{S}_{k}^{(e q)}(t)=0$. 
Thus, equation (11) is the generalisation of FDT2 for systems out of equilibrium, and indeed it possesses the correct limit to the equilibrium FDT2 [3],

$$
\left\langle\mathcal{R}_{-\mathbf{k}}^{(e q)}(\tau, \tau) \mathcal{R}_{\mathbf{k}}^{(e q)}(t, \tau)\right\rangle=\frac{N k^{2}}{\beta m} \gamma_{\mathbf{k}}^{(e q)}(t-\tau)
$$

while $\chi_{\mathbf{k}}^{(e q)}(t, \tau)=0$, and this quantity does not appear in the equilibrium theory.

It is still necessary to determine the generalised frequency, $\Omega_{\mathbf{k}}(t)$, and the instantaneous part of the friction, $\nu_{\mathbf{k}}(t)$, to characterise completely the model. Thus, we write equations (10) for the time $\tau$,

$$
\ddot{\rho}_{\mathbf{k}}(\tau)+\nu_{\mathbf{k}}(\tau) \dot{\rho}_{\mathbf{k}}(\tau)+\Omega_{\mathbf{k}}(\tau) \rho_{\mathbf{k}}(\tau)=\mathcal{R}_{\mathbf{k}}(\tau, \tau)
$$

and we require the following initial conditions for our stochastic process,

$$
\begin{aligned}
\left\langle\rho_{-\mathbf{k}}(\tau) \mathcal{R}_{\mathbf{k}}(\tau, \tau)\right\rangle & =0 \\
\left\langle\dot{\rho}_{-\mathbf{k}}(\tau) \mathcal{R}_{\mathbf{k}}(\tau, \tau)\right\rangle & =0
\end{aligned}
$$

These are consistent with the causality relations of equations $(3,4)$, but one can imagine other models where they are not applied. Their choice leads to the following relations,

$$
\begin{aligned}
& \frac{1}{2} \ddot{S}_{k}(\tau)-J_{k}(\tau)+\frac{1}{2} \nu_{\mathbf{k}}(\tau) \dot{S}_{k}(\tau)+\Omega_{\mathbf{k}}(\tau) S_{k}(\tau)=0 \\
& \frac{1}{2} \dot{J}_{k}(\tau)+\nu_{\mathbf{k}}(\tau) J_{k}(\tau)+\frac{1}{2} \Omega_{\mathbf{k}}(\tau) \dot{S}_{k}(\tau)=0
\end{aligned}
$$

Solutions to these equations are,

$$
\begin{aligned}
\Omega_{\mathbf{k}}(\tau) & =\frac{4 J_{k}^{2}(\tau)-2 J_{k}(\tau) \ddot{S}_{k}(\tau)+\dot{J}_{k}(\tau) \dot{S}_{k}(\tau)}{4 J_{k}(t) S_{k}(\tau)-\dot{S}_{k}^{2}(\tau)} \\
\nu_{\mathbf{k}}(\tau) & =\frac{\ddot{S}_{k}(\tau) \dot{S}_{k}(\tau)-2 \dot{J}_{k}(\tau) S_{k}(\tau)-2 J_{k}(\tau) \dot{S}_{k}(\tau)}{4 J_{k}(\tau) S_{k}(\tau)-\dot{S}_{k}^{2}(\tau)}
\end{aligned}
$$

Note that the choice (9) implies four unknown parameters, and equations $(7,8,23,24)$ fix these in terms of observables, and of the noise distribution, both of which might be considered input into the theory or determined selfconsistently. It is interesting to note that a very similar structure has been 
developed by Latz [9], using the method of non-equilibrium projector operators.

We shall discuss the first attempts to determine these quantities self-consistently within certain approximations.

Following the same kind of steps made in $[2,14]$ we define,

$$
\ddot{\rho}_{\mathbf{k}}(t)+\nu_{\mathbf{k}}(t) \dot{\rho}_{\mathbf{k}}(t)+\Omega_{\mathbf{k}}(t) \rho_{\mathbf{k}}(t)=\mathcal{F}_{\mathbf{k}}(t)
$$

where $\mathcal{F}_{\mathbf{k}}(t)$ are considered to be the couplings between the modes. If we seek to minimize these, then the 'best' choice of modes can be determined by,

$$
\begin{aligned}
& \frac{\partial}{\partial \Omega_{\mathbf{k}}(t)}\left\langle\left|\mathcal{F}_{\mathbf{k}}(t)\right|^{2}\right\rangle=0 \\
& \frac{\partial}{\partial \nu_{\mathbf{k}}(t)}\left\langle\left|\mathcal{F}_{\mathbf{k}}(t)\right|^{2}\right\rangle=0
\end{aligned}
$$

These equations give the same solutions as the ones given in (23) and (24), providing one means of interpreting the choice (19) and (20).

Now we define the normalised correlators,

$$
\Phi_{k}(t, \tau)=\frac{S_{k}(t, \tau)}{S_{k}(\tau, \tau)} ; \quad \Psi_{k}(t, \tau)=\frac{\left\langle\dot{\rho}_{-\mathbf{k}}(\tau) \rho_{\mathbf{k}}(t)\right\rangle}{S_{k}(\tau, \tau)}
$$

and, using the Langevin equations (10) we write,

$$
\begin{aligned}
& \frac{\partial^{2}}{\partial t^{2}} \Phi_{k}(t, \tau)+\nu_{\mathbf{k}}(t) \frac{\partial}{\partial t} \Phi_{k}(t, \tau)+\Omega_{\mathbf{k}}(t) \Phi_{k}(t, \tau) \\
& +\int_{\tau}^{t} d t^{\prime} \chi_{\mathbf{k}}\left(t, t^{\prime}\right) \Phi_{k}\left(\tau, t^{\prime}\right)+\int_{\tau}^{t} d t^{\prime} \gamma_{\mathbf{k}}\left(t, t^{\prime}\right) \frac{\partial}{\partial t^{\prime}} \Phi_{k}\left(\tau, t^{\prime}\right)=0 \\
& \frac{\partial^{2}}{\partial t^{2}} \Psi_{k}(t, \tau)+\nu_{\mathbf{k}}(t) \frac{\partial}{\partial t} \Psi_{k}(t, \tau)+\Omega_{\mathbf{k}}(t) \Psi_{k}(t, \tau) \\
& +\int_{\tau}^{t} d t^{\prime} \chi_{\mathbf{k}}\left(t, t^{\prime}\right) \Psi_{k}\left(\tau, t^{\prime}\right)+\int_{\tau}^{t} d t^{\prime} \gamma_{\mathbf{k}}\left(t, t^{\prime}\right) \frac{\partial}{\partial t^{\prime}} \Psi_{k}\left(\tau, t^{\prime}\right)=0
\end{aligned}
$$

(29) and (30) are the equations of motion for the system. This set of equations is equivalent to the equations proposed in [9]. 
Now, these equations require knowledge only of the dispersion of the noise $\langle\mathcal{R}(t, \tau) \mathcal{R}(\tau, \tau)\rangle$ (see equations $(11,12)$ ), as well as $S_{k}(\tau)$ and $J_{k}(\tau)$.

Before turning to make useful approximations, we discuss briefly a new method that leads to closure of these equations. It can lead to a sequence of corrections, and, in the equilibrium case leads to the known MCT equations within the same type of approximations.

The idea consists in applying a variational principle for that part of the exact random force that is hard to calculate. We shall write a sort of constitutive relation for the noise that connects it to density variables, but where the noise is constrained to have certain reasonable properties, essentially ensuring that both sides of the stochastic equations are consistent. We therefore write,

$$
\begin{aligned}
& \mathcal{R}_{\mathbf{k}}^{V A R}(t, \tau)=\Omega_{\mathbf{k}}(t) \rho_{\mathbf{k}}(t)+\nu_{\mathbf{k}}(t) \dot{\rho}_{\mathbf{k}}(t)+d_{\mathbf{k}}^{(1)}(t) \rho_{\mathbf{k}}(t)+\sum_{k^{\prime} \neq k} d_{\mathbf{k}, \mathbf{k}^{\prime}}^{(2)}(t) \rho_{\mathbf{k}-\mathbf{k}^{\prime}}(t) \rho_{\mathbf{k}^{\prime}}(t) \\
& +\sum_{k^{\prime}, k^{\prime \prime} \neq k^{\prime}} d_{\mathbf{k}, \mathbf{k}^{\prime}, \mathbf{k}^{\prime \prime}}^{(3)}(t) \rho_{\mathbf{k}-\mathbf{k}^{\prime \prime}}(t) \rho_{\mathbf{k}^{\prime \prime}-\mathbf{k}^{\prime}}(t) \rho_{\mathbf{k}^{\prime}}(t)+\ldots \\
& +\int_{\tau}^{t} d t^{\prime} \gamma_{\mathbf{k}}\left(t, t^{\prime}\right) \dot{\rho}_{\mathbf{k}}\left(t^{\prime}\right)+\int_{\tau}^{t} d t^{\prime} \chi_{\mathbf{k}}\left(t, t^{\prime}\right) \rho_{\mathbf{k}}\left(t^{\prime}\right)
\end{aligned}
$$

where the set of parameters $d^{(n)}$ have to be determined by making this trial form the closest possible to the true one, that contains $\ddot{\rho}_{\mathbf{k}}(t)$. In principle, one needs an infinite number of the terms in the sum (31), and the level of the approximation of a theory will be correspondent with the number of terms considered.

To be acceptable this choice of noise must be 'faithful', in the sense that the Langevin process of equations (10) should be preserved. This issue can be partially addressed by requiring that the noise fluctuations on a single time slice are consistent with the Langevin process (10). Thus, we impose,

$$
\frac{\partial}{\partial d^{(n)}(t)}\left\langle\left|\mathcal{R}_{\mathbf{k}}(t, \tau)-\mathcal{R}_{\mathbf{k}}^{V A R}(t, \tau)\right|^{2}\right\rangle=0
$$

where $\mathcal{R}_{\mathbf{k}}(t, \tau)$ is defined in (10). This leads to an infinite set of coupled equaltime equations. In the next section we shall give an example of these.

The other conditions to be satisfied by the expansion (31) of the noise are the constraints $(19,20)$. These conditions can now be explicitly written as,

$$
\Omega_{\mathbf{k}}(\tau) S_{k}(\tau)+\frac{1}{2} \nu_{\mathbf{k}}(\tau) \dot{S}_{k}(\tau)+d_{\mathbf{k}}^{(1)}(\tau) S_{\mathbf{k}}(\tau)+\frac{1}{N} \sum_{k^{\prime} \neq k} d_{\mathbf{k}, \mathbf{k}^{\prime}}^{(2)}(\tau)\left\langle\rho_{-\mathbf{k}}(\tau) \rho_{\mathbf{k}-\mathbf{k}^{\prime}}(\tau) \rho_{\mathbf{k}^{\prime}}(\tau)\right\rangle
$$




$$
\begin{aligned}
& +\frac{1}{N} \sum_{k^{\prime}, k^{\prime \prime} \neq k^{\prime}} d_{\mathbf{k}, \mathbf{k}^{\prime}, \mathbf{k}^{\prime \prime}}^{(3)}(\tau)\left\langle\rho_{-\mathbf{k}}(\tau) \rho_{\mathbf{k}-\mathbf{k}^{\prime \prime}}(t) \rho_{\mathbf{k}^{\prime \prime}-\mathbf{k}^{\prime}}(\tau) \rho_{\mathbf{k}^{\prime}}(\tau)\right\rangle+\ldots=0 \\
& \frac{1}{2} \Omega_{\mathbf{k}}(\tau) \dot{S}_{k}(\tau)+\nu_{\mathbf{k}}(\tau) J_{k}(\tau)+\frac{1}{2} d_{\mathbf{k}}^{(1)}(\tau) \dot{S}_{\mathbf{k}}(\tau)+\frac{1}{N} \sum_{k^{\prime} \neq k} d_{\mathbf{k}, \mathbf{k}^{\prime}}^{(2)}(\tau)\left\langle\dot{\rho}_{-\mathbf{k}}(\tau) \rho_{\mathbf{k}-\mathbf{k}^{\prime}}(\tau) \rho_{\mathbf{k}^{\prime}}(\tau)\right\rangle \\
& +\frac{1}{N} \sum_{k^{\prime}, k^{\prime \prime} \neq k^{\prime}} d_{\mathbf{k}, \mathbf{k}^{\prime}, \mathbf{k}^{\prime \prime}}^{(3)}(\tau)\left\langle\dot{\rho}_{-\mathbf{k}}(\tau) \rho_{\mathbf{k}-\mathbf{k}^{\prime \prime}}(t) \rho_{\mathbf{k}^{\prime \prime}-\mathbf{k}^{\prime}}(\tau) \rho_{\mathbf{k}^{\prime}}(\tau)\right\rangle+\ldots=0
\end{aligned}
$$

These would be exact equations of motion determining the non-equilibrium structure factor and currents, providing one knows the couplings $d^{(n)}$ and the expansion (31) is exact. We now discuss some simple closure ideas. These should not be regarded as complete, but merely indicative of the sorts of approximations that will need to be considered in future.

\section{Simplest Closure}

It is now necessary to employ some approximations regarding the multiple averages involving densities, or equivalently the noise distribution, as well as the kinetic terms. These last terms would be well approximated by their equilibrium limits if we are exploring the system after the velocities have equilibrated, and this is the line of thinking we shall pursue as a first approximation. A different approach has to be taken to account for the multiple averages and also, for the equal time structure factor that is itself evolving in time.

To make relation to the known MCT theory at equilibrium we shall consider only the first non-linear term in the sum which represents the noise (31), and thus, the only parameters left to choose are $d_{\mathbf{k}}^{(1)}(t)$ and $d_{\mathbf{k}, \mathbf{k}^{\prime}}^{(2)}(t)$. We then apply the minimization condition (32),

$$
\begin{aligned}
& \frac{\partial}{\partial d_{\mathbf{k}}^{(1)}(t)}\left\langle\left|\ddot{\rho}_{\mathbf{k}}(t)-d_{\mathbf{k}}^{(1)}(t) \rho_{\mathbf{k}}(t)-\sum_{k^{\prime} \neq k} d_{\mathbf{k}, \mathbf{k}^{\prime}}^{(2)}(t) \rho_{\mathbf{k}-\mathbf{k}^{\prime}}(t) \rho_{\mathbf{k}^{\prime}}(t)\right|^{2}\right\rangle=0 \\
& \frac{\partial}{\partial d_{\mathbf{k}, \mathbf{p}}^{(2)}(t)}\left\langle\left|\ddot{\rho}_{\mathbf{k}}(t)-d_{\mathbf{k}}^{(1)}(t) \rho_{\mathbf{k}}(t)-\sum_{k^{\prime} \neq k} d_{\mathbf{k}, \mathbf{k}^{\prime}}^{(2)}(t) \rho_{\mathbf{k}-\mathbf{k}^{\prime}}(t) \rho_{\mathbf{k}^{\prime}}(t)\right|^{2}\right\rangle=0
\end{aligned}
$$

This explicitly gives the conditions,

$$
\sum_{k^{\prime} \neq k} d_{\mathbf{k}, \mathbf{k}^{\prime}}^{(2)}(t)\left\langle\rho_{-\mathbf{k}}(t) \rho_{\mathbf{k}-\mathbf{k}^{\prime}}(t) \rho_{\mathbf{k}^{\prime}}(t)\right\rangle
$$




$$
\begin{aligned}
& +\mathrm{d}_{\mathbf{k}}^{(1)}(t)\left\langle\rho_{-\mathbf{k}}(t) \rho_{\mathbf{k}}(t)\right\rangle-\left\langle\rho_{-\mathbf{k}}(t) \ddot{\rho}_{\mathbf{k}}(t)\right\rangle=0 \\
& \sum_{k^{\prime} \neq k} d_{\mathbf{k}, \mathbf{k}^{\prime}}^{(2)}(t)\left\langle\rho_{-\mathbf{k}+\mathbf{p}}(t) \rho_{-\mathbf{p}}(t) \rho_{\mathbf{k}-\mathbf{k}^{\prime}}(t) \rho_{\mathbf{k}^{\prime}}(t)\right\rangle \\
& +\mathrm{d}_{\mathbf{k}}^{(1)}(t)\left\langle\rho_{-\mathbf{k}+\mathbf{p}}(t) \rho_{-\mathbf{p}}(t) \rho_{\mathbf{k}}(t)\right\rangle-\left\langle\rho_{-\mathbf{k}+\mathbf{p}}(t) \rho_{-\mathbf{p}}(t) \ddot{\rho}_{\mathbf{k}}(t)\right\rangle=0
\end{aligned}
$$

to solve for finding the best possible coefficients $d_{\mathbf{k}}^{(1)}(t)$ and $d_{\mathbf{k}, \mathbf{k}^{\prime}}^{(2)}(t)$. Equation (37) is trivial, as it corresponds to the same equation as (33).

If we had chosen to work in the Newtons equation representation we would at this point still have the bare potential present in the problem $[2,14]$. In essence, by choosing the best form of the noise in terms of density in an independent manner, as above, we renormalise the instantaneous forces experienced by the density waves at a single time slice, away from what they would have been if we had the bare potential to generate the noise correlations [2,14]. This may also be viewed as performing a partial sum over some of the noise to give exact, or nearly so, equal-time averages, and leaving the averages to be calculated in the remaining correlators to be carried out with the remaining noise. The most important approximations are then due to approximations of the correlations between different time slices. In a manner, we may view this as having renormalised the vertices of the problem prior to proceeding with any approximation of the unequal time correlators.

At this stage we could in principle solve for the coefficients, but consistent with previous MCT approximations, we may further simplify the problem. As in equilibrium MCT [1], we choose a Gaussian approximation for the four point averages and a superposition approximation for the triplets, $\left\langle\rho_{-\mathbf{k}}(t) \rho_{\mathbf{p}}(t) \rho_{\mathbf{q}}(t)\right\rangle \approx$ $N S_{k}(t) S_{p}(t) S_{q}(t) \delta_{\mathbf{k}, \mathbf{p}+\mathbf{q}}$, which consists in neglecting the triple direct correlation function $c_{3}$.

Recall that these averages are not over all the noise ensemble but only that part remaining after the pre-averaging mentioned above. In this sense, using the leading non-trivial approximation for averages (Gaussian for even averages, superposition for odd averages) may not be as severe an approximation as one would think at first sight.

Indeed, in the equilibrium case solutions of equation (38), in these approximations, are given by,

$$
d_{k}^{(1) e q} \approx-\frac{k^{2}}{\beta m S_{k}^{(e q)}} ; \quad d_{\mathbf{k}, \mathbf{k}^{\prime}}^{(2) e q} \approx \frac{\mathbf{k} \cdot \mathbf{k}^{\prime}}{\beta m V} c_{k^{\prime}}^{(e q)}
$$

where $c_{k}^{(e q)}$ is the equilibrium direct correlation function. We have also assumed 
$\sum_{k^{\prime} \neq k} d_{\mathbf{k}, \mathbf{k}^{\prime}}^{(2)(e q)} S_{-\mathbf{k}}^{(e q)} S_{\mathbf{k}-\mathbf{k}^{\prime}}^{(e q)} S_{\mathbf{k}^{\prime}}^{(e q)}=0$.

Thus, inserting this formula in (31) we can calculate (11) neglecting the integral contribution, but still accepting (29) as the correct equations of motions, recovering the well established equilibrium MCT equations [1,14].

In principle, out of equilibrium, it is still possible to solve for the coefficients $d_{\mathbf{k}, \mathbf{k}^{\prime}}^{(2)}(t)$, that minimize (36), but to have some explicit expressions for them we have to make further approximations than those in the equilibrium case. We shall, as a first approximation, consider ourselves to be in the regime where the kinetic contributions to the observables are the same as in the equilibrium limit, and we thereby assume that the velocities relax towards their equilibrium values much faster than the positions.

In the following we consider the case of any time instant $t^{\prime}$, in the range between $\tau$ and $t$. Thus, we approximate,

$$
\left\langle\ddot{\rho}_{-\mathbf{k}}\left(t^{\prime}\right) \rho_{\mathbf{k}-\mathbf{p}}\left(t^{\prime}\right) \rho_{\mathbf{p}}\left(t^{\prime}\right)\right\rangle \approx-\frac{N \mathbf{k} \cdot(\mathbf{k}-\mathbf{p})}{\beta m} S_{p}\left(t^{\prime}\right)-\frac{N \mathbf{k} \cdot \mathbf{p}}{\beta m} S_{|\mathbf{k}-\mathbf{p}|}\left(t^{\prime}\right) .
$$

The last approximation to be made is that $\left\langle\rho_{-\mathbf{k}}\left(t^{\prime}\right) \ddot{\rho}_{\mathbf{k}}\left(t^{\prime}\right)\right\rangle=(1 / 2) \ddot{S}_{k}\left(t^{\prime}\right)-$ $J_{k}\left(t^{\prime}\right) \approx-k^{2} / \beta m$, with $\ddot{S}_{k}\left(t^{\prime}\right) \approx 0$.

We can now write the result for the best coefficients in this approximations,

$$
d_{k}^{(1)}\left(t^{\prime}\right) \approx-\frac{k^{2}}{\beta m S_{k}\left(t^{\prime}\right)} ; \quad d_{\mathbf{k}, \mathbf{k}^{\prime}}^{(2)}\left(t^{\prime}\right) \approx \frac{\mathbf{k} \cdot \mathbf{k}^{\prime}}{\beta m V} c_{k^{\prime}}\left(t^{\prime}\right)
$$

with $n c_{k}\left(t^{\prime}\right)=1-1 / S_{k}\left(t^{\prime}\right)$ the generalised direct correlation function, with $n$ the number density $N / V$. Evidently, this is a sort of adiabatic approximation, in which, ultimately, we expect changes in the noise distribution to arise from changes in the slowly evolving structure.

We now use (10) and (31) to obtain,

$$
\begin{aligned}
\mathcal{R}_{\mathbf{k}}\left(t^{\prime}, \tau\right) & \approx \frac{1}{\beta m V} \sum_{\mathbf{k}^{\prime} \neq \mathbf{k}}\left(\mathbf{k} \cdot \mathbf{k}^{\prime}\right) c_{k^{\prime}}\left(t^{\prime}\right) \rho_{\mathbf{k}-\mathbf{k}^{\prime}}\left(t^{\prime}\right) \rho_{\mathbf{k}^{\prime}}\left(t^{\prime}\right)+\nu_{\mathbf{k}}(t) \dot{\rho}_{\mathbf{k}}(t) \\
& +\int_{\tau}^{t} d t^{\prime} \gamma_{\mathbf{k}}\left(t, t^{\prime}\right) \dot{\rho}_{\mathbf{k}}\left(t^{\prime}\right)+\int_{\tau}^{t} d t^{\prime} \chi_{\mathbf{k}}\left(t, t^{\prime}\right) \rho_{\mathbf{k}}\left(t^{\prime}\right)
\end{aligned}
$$

where $t^{\prime}$ can be any instant between $\tau$ and $t$. The term containing the memory kernel is neglected in equilibrium MCT $[1,14]$. We will, therefore, also neglect 
the two integral terms, which in any case would be of higher order in our arguments.

Now, if we approximate the four-point density correlation for different times, by Gaussian decomposition, as before, and we neglect the contributions arising from the term $\nu_{\mathbf{k}}(t) \dot{\rho}_{\mathbf{k}}(t)$ in (42) because of higher order, we finally find the generalised expression for the memory kernel $\gamma_{\mathbf{k}}(t, \tau)$,

$$
\begin{aligned}
& \gamma_{\mathbf{k}}(t, \tau) \approx \frac{4 N S_{k}(\tau)}{\beta^{2} m^{2} V^{2}\left[4 J_{k}(\tau) S_{k}(\tau)-\dot{S}_{k}^{2}(\tau)\right]} \sum_{\mathbf{k}^{\prime} \neq \mathbf{k}}\left[\left(\mathbf{k} \cdot \mathbf{k}^{\prime}\right)^{2} c_{k^{\prime}}(\tau) c_{k^{\prime}}(t)\right. \\
& \left.+\left(\mathbf{k} \cdot \mathbf{k}^{\prime}\right) \mathbf{k} \cdot\left(\mathbf{k}-\mathbf{k}^{\prime}\right) c_{k^{\prime}}(\tau) c_{\left|\mathbf{k}-\mathbf{k}^{\prime}\right|}(t)\right] S_{\left|\mathbf{k}-\mathbf{k}^{\prime}\right|}(t, \tau) S_{k^{\prime}}(t, \tau) .
\end{aligned}
$$

and the memory kernel $\chi_{\mathbf{k}}(t, \tau)$ is then obtained combining this expression with (12). We can now apply successive levels of approximation to equation $(33,34)$ to find an equation of motion that determines the non-equilibrium structure factor, $S_{\mathbf{k}}(t)$. In this way, we have a simple theory of the nonequilibrium dynamical structure factor.

\section{Conclusions}

In this paper we have derived a generalised Langevin equation that should reasonably describe an out-of-equilibrium dynamically slowed system. This leads to new constraints on the noise distributions rather than traditional FDT2, but these constraints are still practicable to apply. We then expand the noise in a 'Landau type' expansion of the density variables, leading to a non-linear generalised Langevin process. However we conceive that some preaveraging of the variables has taken place so that exact equal time averages are recovered to a high level of quality by assuming remaining noise is only gaussian distributed. To preserve fidelity of the Langevin process we then insist that the noise be a faithful representation, and that the structure of the Langevin process is still preserved. These requirements are implemented by a variational principle to determine the renormalised coefficients of the noise, and by implementing the orthogonality of the noise to the 'slow' variables, $\rho_{\mathbf{k}}(t)$ and $\dot{\rho}_{\mathbf{k}}(t)$. This leads in principle to the determination of all of the unknown coefficients of the noise.

Further approximations are possible but not obligatory, but they make the contact with existing ideas easier. The outcome is that we end up with a theory that must be considered as an 'adiabatic' extension of the existing MCT in the sense that kinetic terms are neglected, assumed to be equilibrated. The MCT equations are therefore modified, but the form of the memory kernel is preserved, only it now includes the dependence on the waiting time. In 
equilibrium MCT one typically inputs any good approximation to the static structure factor for which the YBG (Yvon-Born-Green Equation) equation of constraint should hold, or hold approximately[2]. Here we deduce the corresponding conditions, and these generate an equation of motion for the equal time structure factor and current correlator. These equations would be exact providing the expansion chosen for the random force, as in (31), is a complete one. As non-equilibrium structure factors to use as inputs are much less known than equilibrium ones, these equations, or some similar ones, will be crucial to close the theory.

The strategy we have pursued here seems different to that developed in [9], and it is as yet too early to see how these different approaches will relate to each other. Probably the answer to that question is that in the end, as with the equilibrium MCT case [14], a fair degree of correspondence will emerge, though it will be important to see if either approach leads naturally to higher levels of approximation.

From what we know about colloidal systems near their kinetic arrest, this theory should be reasonably successful in describing the phenomena qualitatively, perhaps, as with equilibrium MCT, quantitatively. This opens the possibility to begin systematic study of the aging of colloids and soft materials $[16,17]$. However, to make useful progress, in the regime where FDT2 is violated for molecular glasses, it will require better approximations than those shown here, and this is a matter to which we, and others, most certainly will direct our attentions.

\section{Acknowledgements}

The authors wish to note the most interesting discussions with A. Latz, for which they are most grateful. They also acknowledge very useful remarks made by W. Götze, and discussions with K. Kawasaki, A. Crisanti and G. Parisi. F.S. and P.T. are supported by INFM-PRA-HOP and MURST-COFIN2000. Both Dublin and Rome groups are supported by COST P1.

\section{References}

[1] U. Bengtzelius, W. Götze and A. Sjölander, J. Phys. C 17, 5915 (1984); W. Götze, in Liquids, Freezing and Glass Transition, edited by J.P. Hansen, D.Levesque and J.Zinn-Justin (North-Holland, Amsterdam, 1991).

[2] E. Zaccarelli, G. Foffi, F. Sciortino, P. Tartaglia and K. A. Dawson, Europhysics Letts, 55, 139 (2001). 
[3] R. Kubo, M. Toda and N. Hashitsume, Statistical Physics II, Springer-Verlag, Berlin (1991), 2nd Edition.

[4] W. van Megen, S. M. Underwood and P. N. Pusey, Phys. Rev. Lett. 67, 1586 (1991); W. van M. and S. M. U., Phys. Rev. Lett. 70, 2766 (1993) and 72, 1773 (1994).

[5] J-P. Bouchad, L. F. Cugliandolo, J. Kurchan and M. Mezard, Physica A 226, 243 (1996).

[6] W. Kob and J.-L. Barrat, Phys. Rev. Lett. 78, 4581 (1997).

[7] G. Parisi, Phys. Rev. Lett. 79, 3660 (1997).

[8] F. Sciortino and P. Tartaglia, Phys. Rev. Lett. 86, 107 (2001).

[9] A. Latz, J. Phys. Cond. Matt. 12, 6353 (2000); cond-mat/0106086.

[10] T. R. Kirkpatrick and D. Thirumalai, J. Phys. A22, L119 (1989).

[11] J-P. Bouchad and M. Mezard, J. Phys. I (France) 4, 1109 (1991); E. Marinari, G. Parisi and F. Ritort, J. Phys. A27, 7615 (1991).

[12] L. F. Cugliandolo, J. Kurchan, G. Parisi and F. Ritort, Phys. Rev. Lett. 74, 1012 (1995); P. Chandra, L. Ioffe and D. Sherrington, Phys. Rev. Lett. 75, 713 (1995).

[13] L. F. Cugliandolo and J. Kurchan, Phys. Rev. Lett. 71, 173 (1993); J. Phys. A27, 5719 (1991).

[14] E. Zaccarelli, P. De Gregorio, G. Foffi, F. Sciortino, P. Tartaglia and K. A. Dawson, unpublished.

[15] W.Götze, J. Phys. Cond. Matt. 11, A1 (1999).

[16] W. van Megen, T. C. Mortensen, S. R. Williams and J. Müller, Phys. Rev. E 58, 6073 (1998).

[17] L. Cipelletti, S. Manley, R. C. Ball and D. A. Weitz, Phys. Rev. Lett. 84, 2275 (2000). 